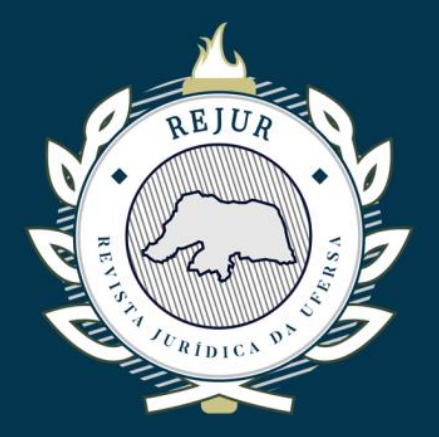

\title{
LA SEGURIDAD DE LOS ORGANISMOS MODIFICADOS GENÉTICAMENTE EN EL ÁMBITO ALIMENTARIO: PRINCIPIOS FUNDAMENTALES
}

SAFETY OF GENETICALLY MODIFIED ORGANISMS IN THE FOOD SECTOR: FUNDAMENTAL PRINCIPLES

\section{LORENA GALLARDO*}

RESUMO

El presente trabajo procura brindar una relación sucinta de los principios jurídicos más elementales vinculados al proceso de evaluación de la seguridad de los organismos modificados genéticamente en el ámbito alimentario, exponiendo sus características más salientes $y$, asimismo, evidenciando las deficiencias que algunos de ellos traen aparejadas en su ejecución.

Palavras-chave: OMG; riesgos; biotecnología.
ABSTRACT

This paper aims to provide a concise exposition of some of the most basic legal principles linked to the process of evaluation of genetically modified food safety, revealing their most salient features and also highlighting the deficiencies that some of them bring along in their application to the products under study.

Keywords: GMO; risks; biotechnology.

* Doutora e Mestra em Derecho Privado Patrimonial pela Universidad de Salamanca (Espanha). Professora Adjunta da Universidad Nacional del Nordeste (Argentina). Advogada.

lorenamazz@yahoo.com.ar

Recebido em 26-10-2017 | Aprovado em 26-10-2017

${ }^{1}$ Artigo convidado. Publicação original: Ars luris Salmanticensis, Salamanca, jun./2017, 5 (1), p. 163-187. 


\section{SUMÁRIO}

GENERALIDADES; 1 LOS PRINCIPIOS DE EVALUACIÓN DE RIESGOS Y CASO POR CASO; 2 EL PRINCIPIO DE EQUIVALENCIA SUSTANCIAL; 3 EL PRINCIPIO DE SEGUIMIENTO O TRAZABILIDAD; 3.1 En relación con el etiquetado; 4 EL PRINCIPIO DE PRECAUCIÓN; 4.1 Materialización del Principio de Precaución; 4.2 El Principio de Precaución en la UE; 4.3 El Principio de Precaución en España; 5 EL PRINCIPIO DE PREVENCIÓN; CONSIDERACIONES FINALES.

\section{GENERALIDADES}

El vertiginoso desarrollo de la ciencia y la tecnología hizo necesaria una nueva construcción del riesgo. Los denominados nuevos riesgos se caracterizan por constituir amenazas imperceptibles hasta el momento de su materialización, generalmente efectivizadas luego de períodos de latencia indeterminados. Así, resulta inadecuado pretender observar y analizar estas nuevas situaciones bajo la lupa tradicional del riesgo cuantificable, aplicable a un riesgo objetivo, previsible y calculable ${ }^{2}$. Esta nueva construcción del riesgo se asienta, por el contrario, sobre la idea de indeterminación del conocimiento científico, y presenta como rasgo característico la noción de precaución. En este contexto, hemos identificado a la producción y comercialización de organismos modificados genéticamente ${ }^{3}$ (productos que se caracterizan por la inexistencia de limitaciones en cuanto a los cruzamientos realizados entre individuos de diversa especie, lo que implicaría un salto de las barreras naturalmente establecidas entre las distintas especies que componen el ecosistema) como una actividad propicia para la generación de estos nuevos riesgos.

A efectos de brindar una adecuada protección a los consumidores, la Directiva 2001/95/CE relativa a la seguridad general de los productos, establece que resulta necesario edificar una "legislación comunitaria horizontal que introduzca una obligación general de seguridad de los productos, así como disposiciones sobre las obligaciones generales de productores y distribuidores, sobre el control de la aplicación de los requisitos comunitarios relativos a la seguridad de los productos y sobre el intercambio rápido de información y la actuación a escala comunitaria en determinados casos" (Considerando 4).

En cuanto a la evaluación del riesgo, la comercialización de alimentos o piensos ${ }^{4}$ modificados genéticamente requiere una autorización que se obtiene a posteriori de la realización de un proceso de evaluación que en la UE lleva a cabo la AESA ${ }^{5}$.

\footnotetext{
${ }^{2}$ CARR, S. e IBARRA, A. Las construcciones del riesgo. En LUJÁN, J. L. y ECHEVERRÍA, J., (Eds.): Gobernar los riesgos. Ciencia y valores en la sociedad del riesgo. Madrid: Ed. Biblioteca Nueva, 61, 2009.

${ }^{3}$ En adelante OMG.

${ }^{4}$ Art. 13 - Reglamento (CE) № 178/2002 - "Se entiende por pienso cualquier sustancia o producto, incluidos los aditivos, destinado a la alimentación por vía oral de los animales, tanto si ha sido transformado entera o parcialmente como si no."

${ }^{5}$ El Parlamento Europeo y el Consejo crearon la Autoridad Europea de Seguridad Alimentaria (AESA) en 2002, tras una serie de situaciones de riesgo alimentario que dejaron patente la necesidad de someter el sistema europeo a una revisión profunda, con vistas al desarrollo de una legislación en materia alimentaria. EFSA. 2008:
} 
La autorización, cuando es concedida, significa que el producto cumple con los estándares de seguridad exigidos: no tener efectos negativos sobre la salud humana, la sanidad animal o el medio ambiente; no diferenciarse de los alimentos que está destinado a sustituir, de modo que su consumo habitual resulte desventajoso desde el punto de vista nutricional para los consumidores; y no inducir al consumidor a error.

A nuestro juicio, no obstante, las evaluaciones y estudios actualmente exigidos para la concesión de autorizaciones para la producción y comercialización de OMG resultan, quizás, insuficientes para acreditar la inocuidad de los mismos, ya que no permiten descartar la presencia de riesgos derivados de la manipulación de genes entre organismos de distinta especie que otros estudios realizados de manera independiente confirman.

A pesar de las prescripciones normativas existentes, la complejidad del mundo actual demuestra que se requiere algo más que leyes, que ninguna ley ni código, por minucioso y casuístico que sea, puede prever ni dar solución a los innumerables casos que la práctica ofrece; la vida se renueva sin cesar y se presentan diferentes necesidades que no caben dentro de la fórmula rígida e inflexible de la ley ${ }^{6}$, por lo cual se hace imprescindible a los tribunales recurrir a criterios directivos o pautas orientadoras, a fin de hallar la solución más justa posible.

Por este motivo resulta siempre preciso que, junto a la legislación específica, se enfoque el análisis de las problemáticas bajo tratamiento (en este caso el proceso de evaluación de la seguridad de los OMG alimentarios) también desde los principios involucrados los que, indudablemente, determinarán el rumbo de los procedimientos implicados.

\section{LOS PRINCIPIOS DE EVALUACIÓN DE RIESGOS Y CASO POR CASO}

Uno de los pilares esenciales de la normativa sobre utilización confinada, liberación intencional y comercialización de OMG, así como de la disciplina sobre seguridad alimentaria, es la evaluación de los riesgos que tales organismos puedan provocar en la salud humana o animal y en el medio ambiente ${ }^{7}$.

En la práctica, este principio se traduce en la exigencia de evaluación previa de los riesgos eventuales de todo OMG que pretenda obtener la autorización para su comercialización en el mercado.

Legislativamente, se encuentra receptado por el Reglamento 178/2002/CE ${ }^{8}$, que en su Considerando 16 estableció que "las medidas que adopten los Estados miembros o la Comunidad con respecto a los alimentos y los piensos deben estar basadas, en general, en un

Plan Estratégico de la Autoridad Europea de Seguridad Alimentaria para 2009-2013, Parma, http://www.efsa.europa.eu/en/corporate/doc/stratplan09es.pdf [6 de febrero de 2014].

${ }^{6}$ DIAZ COUSELO, J. M. Los Principios Generales del Derecho. Buenos Aires: Ed. Plus Ultra, 11-17, 1971.

${ }^{7}$ AMAT LLOMBART, P. Derecho de la Biotecnología y los Transgénicos (Especial referencia al sector agrario y alimentario). Valencia: Tirant lo Blanch, 98, 2008.

${ }^{8}$ REGLAMENTO (CE) No 178/2002 DEL PARLAMENTO EUROPEO Y DEL CONSEJO de 28 de enero de 2002 por el que se establecen los principios y los requisitos generales de la legislación alimentaria, se crea la Autoridad Europea de Seguridad Alimentaria y se fijan procedimientos relativos a la seguridad alimentaria. 
análisis de riesgo [...]"9 $\mathrm{y}$, asimismo, el Art. 6 del mismo cuerpo legal, relativo al análisis del riesgo, dispuso que la legislación alimentaria se basará en el análisis del riesgo, con el fin último de lograr un nivel elevado de protección de la salud y la vida de las personas ${ }^{10}$.

A su vez, en España, la Ley $9 / 2003^{11}$, en su Art 6.1 estableció que las actividades se clasificarán en función de la evaluación previa de los riesgos para la salud humana y el medio ambiente, en actividades de riesgo nulo o insignificante, de bajo riesgo, de riesgo moderado y de alto riesgo.

Por su parte, la Ley $17 / 2011$ de 5 de julio sobre seguridad alimentaria y nutrición, establece en su Art. 5 que la legislación alimentaria se basará en el análisis del riesgo, el que se centrará en las pruebas científicas disponibles y se efectuará de manera objetiva y transparente.

El proceso de evaluación del riesgo se compone de cuatro fases, a saber:

1. La identificación del peligro: consistente en la determinación de agentes biológicos o químicos capaces de producir efectos perjudiciales para la salud humana o para el medio ambiente.

2. La caracterización del peligro: que implica la evaluación cualitativa o cuantitativa del efecto dañoso real o supuesto sobre la salud o el medioambiente.

3. La evaluación de la exposición: mediante el examen cualitativo o cuantitativo de eventual ingestión de alimentos dañosos.

4. La caracterización del riesgo: estimación cualitativa y cuantitativa, tomando en cuenta la incertidumbre inherente a las etapas anteriores, la probabilidad de frecuencia y la gravedad de los efectos negativos y de los daños conocidos y potenciales ${ }^{12}$.

Este principio se encuentra estrechamente ligado al principio caso por caso en su ejecución, ya que las evaluaciones son realizadas de manera individualizada para cada

\footnotetext{
${ }^{9}$ Considerando 16 - Reglamento (CE) No 178/2002: "Las medidas que adopten los Estados miembros o la Comunidad con respecto a los alimentos y los piensos deben estar basadas, en general, en un análisis de riesgo, salvo que no se considere adecuado por las circunstancias o la naturaleza de la medida. El recurso a este instrumento antes de adoptar ninguna medida debe evitar que se creen barreras injustificadas a la libre circulación de productos alimenticios."

${ }^{10}$ Art. № 6 - Reglamento (CE) No 178/2002: "1. Con el fin de lograr el objetivo general de un nivel elevado de protección de la salud y la vida de las personas, la legislación alimentaria se basará en el análisis del riesgo, salvo que esto no convenga a las circunstancias o la naturaleza de la medida legislativa. 2. La determinación del riesgo se basará en las pruebas científicas disponibles y se efectuará de una manera independiente, objetiva y transparente."

${ }^{11}$ La Ley 9/2003 de 25 de abril por la que se establece el régimen jurídico de la utilización confinada, liberación voluntaria y comercialización de organismos modificados genéticamente, regula los procesos de autorización de OMG únicamente para el cultivo, la liberación intencional en el medio ambiente o la comercialización en un ámbito distinto al alimentario. Si el operador industrial pretende una autorización alimentaria debe iniciar el trámite conforme al Reglamento 1829/2003 sobre alimentos y piensos modificados genéticamente. DE ROMÁN PÉREZ, R. Organismos modificados genéticamente en el marco del desarrollo rural sostenible. En VATTIER FUENZALIDA, C. (Dir.) y DE ROMÁN PÉREZ, R. (Coord.), El Desarrollo Sostenible en el Ámbito Rural. Navarra: Thomson Reuters, 340-341, 2009.

${ }^{12}$ DRNAS DE CLEMENT Z. El principio de precaución ambiental. La práctica argentina. Córdoba: Lerner Editora, 63- 64, 2008.
} 
operación, es decir, se procura determinar para cada producto u organismo analizado singularmente cuál es su eventual incidencia nociva.

El principio caso por caso es necesario debido a la gran variedad de supuestos, la limitada experiencia actual en la materia y la dificultad que implica el intento de evaluar correctamente riesgos potenciales.

Así lo estableció ya la Directiva 90/219/CEE ${ }^{13}$, considerando que la naturaleza precisa y los riesgos asociados a los microorganismos modificados genéticamente no se conocían aún de modo completo y que el riesgo que suponen debía estudiarse caso por caso ${ }^{14}$.

También fue adoptado por la Directiva 2001/18/CE ${ }^{15}$, en la cual se manifestó la necesidad de establecer procedimientos y criterios armonizados para la evaluación caso por caso de los riesgos potenciales derivados de la liberación intencional de OMG en el medio ambiente ${ }^{16}$.

En España, la Exposición de Motivos de la Ley 9/2003 lo incluye como uno de los principios inspiradores de la misma, definiéndolo como "la evaluación de los riesgos asociados a los organismos modificados genéticamente para cada uno de ellos".

Por ello se concluye que, actualmente, los OMG no están ni prohibidos ni permitidos con carácter general, sino que la decisión la tomarán las autoridades competentes caso a caso, en función del riesgo que cada uno de ellos (y en cada una de las finalidades a que puedan ser destinados) represente para los dos grandes bienes jurídicos protegidos, la salud de las personas y el medio ambiente. Esta decisión no será arbitraria, sino que deberá basarse en la previa evaluación del riesgo que el organismo en cuestión suponga para ambos bienes jurídicos, evaluación que habrá de ser realizada por los particulares interesados con arreglo a criterios estrictamente científicos ${ }^{17}$.

\section{EL PRINCIPIO DE EQUIVALENCIA SUSTANCIAL}

Como ya hemos apuntado, para que un OMG alimentario pueda ser liberado en un mercado determinado, debe ser sometido a rigurosas evaluaciones ante las autoridades de cada país, para lo cual se reúne vasta información relativa al producto obtenida a través de diversos mecanismos. En este sentido, la técnica más generalizada para determinar el grado

\footnotetext{
${ }^{13}$ Directiva 90/219/CEE del Consejo de 23 de abril de 1990 relativa a la utilización confinada de microorganismos modificados genéticamente.

${ }^{14}$ Considerando 7 - Directiva 90/219/CEE del Consejo de 23 de abril de 1990 relativa a la utilización confinada de microorganismos modificados genéticamente: “Considerando que la naturaleza precisa y la escala de los riesgos asociados a los microorganismos modificados genéticamente no se conocen aún de modo completo y que el riesgo que suponen debe estudiarse caso por caso [...]".

15 Directiva 2001/18/CE del Parlamento Europeo y del Consejo, de 12 de marzo de 2001, sobre la liberación intencional en el medio ambiente de organismos modificados genéticamente y por la que se deroga la Directiva 90/220/CEE del Consejo.

${ }^{16}$ Considerando 18 - Directiva 2001/18/CE del Parlamento Europeo y del Consejo de 12 de marzo de 2001 sobre la liberación intencional en el medio ambiente de organismos modificados genéticamente: "Es necesario establecer procedimientos y criterios armonizados para la evaluación caso por caso de los riesgos potenciales derivados de la liberación intencional de OMG en el medio ambiente."

${ }^{17}$ AMAT LLOMBART, P. Op. cit., 104.
} 
de seguridad de esta clase de alimentos se realiza a través de la comparación con sus homólogos convencionales, dado que se parte de la idea de que los productos naturales ofrecen un estándar de seguridad y riesgo mínimo adoptados como aceptables por la sociedad. Esta evaluación de seguridad es lo que la $\mathrm{OCDE}^{18}$ ha dado en Ilamar Principio de Equivalencia Sustancial.

El concepto de Equivalencia Sustancial ${ }^{19}$, según la OCDE, la FAO y la OMS, supone que si un alimento o componente alimenticio nuevo es sustancialmente equivalente a un alimento o componente alimenticio ya existente, puede ser tratado de la misma manera con respecto a la seguridad. Es decir, el resultado de la operatividad de este principio, cuando es positivo, equipara productos de origen agropecuario tradicional a los modificados genéticamente, al confirmar que estos últimos no comportan ningún riesgo adverso adicional.

Este procedimiento no debería constituir por sí mismo una evaluación de inocuidad del alimento, sino sólo su punto de partida ${ }^{20}$. Se recurre a él, concretamente, para determinar similitudes y diferencias entre el alimento nuevo y su homólogo convencional, y así detectar problemas nutricionales, por ejemplo. No se debería pretender, por lo tanto, determinar mediante él la seguridad del nuevo producto de forma absoluta.

Ahora bien, este proceso de evaluación de riesgos, que tiene por objeto estimar el impacto que los efectos intencionales y no intencionales del cambio genético pudieran tener sobre la salud de la población y también sobre el medio ambiente, no se limita a una mera comparación de aspectos superficiales entre ambos organismos, sino que el mismo indaga en factores tales como rasgos fenotípicos y agronómicos de la composición del nuevo producto, proceso de transformación, nuevos rasgos introducidos, posibles efectos secundarios, etc.

Los sectores partidarios de la producción y comercialización de estos organismos cuestionan la exigencia del etiquetado especial de los mismos, con basamento, justamente, en este Principio de Equivalencia Sustancial. Sostienen que este requisito legal debería limitarse sólo a aquellos productos modificados genéticamente que no presentasen dicha equivalencia.

Sin embargo, los colectivos opositores, con sustento en el mal de la Encefalopatía Espongiforme Bovina (conocida como Enfermedad de las Vacas Locas), alegan la inefectividad del Principio de Equivalencia Sustancial para determinar de manera suficiente y acabada el

\footnotetext{
${ }^{18}$ La OCDE (Organización para la Cooperación y el Desarrollo Económicos) agrupa a 34 países miembros y su misión es promover políticas que mejoren el bienestar económico y social de las personas alrededor del mundo. 19 "El concepto de equivalencia sustancial fue desarrollado antes de que cualquiera de los alimentos modificados genéticamente fuera lanzado al mercado. Fue inicialmente descripto en 1993 en un documento titulado "Evaluación sobre la seguridad de los alimentos derivados de la biotecnología moderna: Conceptos y Principios", elaborado por 60 expertos de 19 países pertenecientes a la OCDE que discutieron por más de dos años cómo evaluar los organismos genéticamente mejorados. La mayoría de estos expertos, todos nominados por sus gobiernos, fueron científicos reguladores de agencias de gobierno y ministerios responsables de la seguridad de los consumidores. La evaluación de equivalencia sustancial debe demostrar que las variedades genéticamente mejoradas son tan seguras como sus contrapartes tradicionales, a través de una consideración de los efectos deseados e indeseados de ambas especies." ARGENBIO - CONSEJO ARGENTINO PARA LA INFORMACIÓN Y EL DESARROLLO DE LA BIOTECNOLOGÍA. (s.f.): “Alimentos transgénicos, ¿deberían etiquetarse?”. Cuaderno № 14. Disponible en: http://www.porquebiotecnologia.com.ar/index.php?action=cuaderno\&opt=5\&tipo=1\&note=14. Acesso en: 25 may. 2010.

20 FAO. Evaluación de la inocuidad de los alimentos genéticamente modificados. Instrumentos para capacitadores, Roma, 11., 2009. Disponible en: http://www.fao.org/3/a-i0110s.pdf. Acesso en: 19 de mar. 2015.
} 
carácter de inofensivos de los OMG, explicando que, en el caso citado, las proteínas infecciosas habrían sido idénticas en su secuencia de aminoácidos a las no patológicas, variando sólo en su disposición espacial. Según el Principio de Equivalencia Sustancial, en este caso, un organismo infectado sería idéntico a un organismo sano.

En esta misma línea, la UE ha manifestado que "no es plausible alegar que los productos modificados genéticamente son equivalentes a los productos no modificados genéticamente o que deben ser tratados como estos últimos" 21 .

La UE lo receptó inicialmente en la Directiva 90/220/CEE ${ }^{22}$, al establecer que "los alimentos cuyo valor nutricional, composición y uso, fueran reconocidos como sustancialmente equivalentes a los ya existentes, no necesitaban consentimiento sino sólo notificación a las autoridades públicas" 23 .

En torno a los OMG, la cuestión se clarificó a partir del Reglamento sobre alimentos nuevos 258/97, el cual estableció un procedimiento simplificado para la aprobación en los supuestos en que los alimentos fueran sustancialmente equivalentes al alimento ya existente. Sin embargo, las organizaciones de consumidores lo criticaron como base inadecuada para la determinación de la seguridad alimentaria. Las protestas llevaron a la UE a reconsiderar sus propios procedimientos, llegando las controversias acerca de la cuestión a conocimiento del TJCE, el cual "sugirió la necesidad de una evidencia científica mayor para demostrar la equivalencia en la composición de los alimentos MG o para probar los efectos toxicológicos aparentemente equivalentes" 24 .

Finalmente, el Reglamento $1829 / 2003^{25}$ terminó por rechazar el Principio de Equivalencia Sustancial como criterio apropiado para evaluar la seguridad de los alimentos modificados genéticamente.

Sin embargo, resulta preciso mencionar que el Principio de Equivalencia Sustancial continúa siendo el estándar de evaluación de seguridad de los alimentos modificados genéticamente en los Estados Unidos ${ }^{26}$.

\footnotetext{
${ }^{21}$ OMC, COMUNIDADES EUROPEAS. Medidas que afectan a la aprobación y comercialización de productos biotecnológicos. Informes del Grupo Especial. Reclamaciones WT/DS291/R WT/DS292/R WT/DS293/R, Primera Comunicación escrita de las Comunidades Europeas.

22 Directiva 90/220/CEE del Consejo, de 23 de abril de 1990, sobre la liberación intencional en el medio ambiente de organismos modificados genéticamente.

${ }^{23}$ FERNÁNDEZ DÍEZ, M. C. y CORRIPIO GIL - DELGADO, M. R. Los conflictos en la política comercial de la Unión Europea: el caso de la aplicación del Principio de Precaución en la normativa comunitaria de Bioseguridad. En DE TOMÁS MORALES, S. y VAQUERO LAFUENTE, E., El día de Europa: La Europa de los 25. Madrid: Universidad Pontificia Comillas, 73, 2005.

24 Ibídem, p. 75.

${ }^{25}$ REGLAMENTO (CE) No 1829/2003 DEL PARLAMENTO EUROPEO Y DEL CONSEJO de 22 de septiembre de 2003 sobre alimentos y piensos modificados genéticamente.

26 "La FDA no obliga a que el salmón AquAdvantage sea etiquetado como transgénico, ya que "es tan seguro y nutritivo como el salmón atlántico no modificado genéticamente" y "no es materialmente diferente". EL PAÍs. Aprobado el primer animal transgénico para consumo humano. Disponible en: http://elpais.com/elpais/2015/11/19/ciencia/1447945426_325310.html. Acesso en: 21 nov. 2015.
} 


\section{EL PRINCIPIO DE SEGUIMIENTO O TRAZABILIDAD}

Queda claro que el Estado está obligado a tomar todas las precauciones adecuadas para minimizar el riesgo de que los bienes fundamentales de la sociedad sufran cualquier daño, por más improbable que éste pueda parecer. Pero también es razonable admitir que el riesgo cero, la seguridad absoluta, es inalcanzable.

A la circunstancia de que nadie está en condiciones de garantizar con total seguridad que un bien no vaya a sufrir menoscabo alguno, se añade la necesidad de tolerar un cierto nivel de riesgo con el objeto de satisfacer otros fines constitucionalmente relevantes, como la libertad ${ }^{27}$.

Es inherente a toda regulación jurídica su provisionalidad, ya que el Derecho es un sistema de normas en permanente evolución. En el caso de la protección contra riesgos vinculados a los OMG, esta característica se halla acentuada, ya que si bien la evaluación de los riesgos en este ámbito se rige por criterios rigurosos de análisis previo a su libre introducción en los mercados, no se puede desconocer que en estos casos y dada la naturaleza del producto en cuestión, la Administración actúa en una situación de gran incertidumbre, lo que genera desconfianza respecto al posterior desarrollo y materialización de los riesgos vinculados a determinados productos modificados genéticamente, en un mediano o largo plazo.

Es en este orden de ideas donde opera el principio de trazabilidad, ya que cuanto menor es la información disponible, y por ende, mayor la incertidumbre acerca de las consecuencias que determinados hechos pudiesen acarrear, más fácil es que con el devenir del tiempo surjan nuevos datos que aporten soluciones diversas y muchas veces determinen que la regulación establecida es inadecuada. Es por ello comprensible que la provisionalidad y la posibilidad de revisión sean connaturales a las decisiones de riesgo, tanto más cuanto mayor sea la inseguridad en la que los poderes públicos se vean abocados a decidir ${ }^{28}$.

La finalidad práctica del principio consiste en seguir de cerca el desarrollo y uso de los OMG, proceder al control de los posibles efectos acumulados a largo plazo y detectar e identificar cualquier efecto directo, indirecto, inmediato, diferido o imprevisto que los productos que contengan o consistan en OMG puedan producir a la salud humana después de su comercialización ${ }^{29}$.

A fin de cumplimentar las prescripciones de tal principio (contenido en la Directiva 2001/18/CE), que considera necesario establecer el control continuado de los OMG tras su liberación deliberada o su comercialización como productos o componentes de productos, se prevé la puesta en funcionamiento de un plan de seguimiento de los mismos por parte de los Estados miembros.

\footnotetext{
${ }^{27}$ DOMÉNECH PASCUAL, G. Derechos Fundamentales y riesgos tecnológicos. Madrid: Centro de Estudios Políticos y Constitucionales, 313-314, 2006.

28 Ibidem, p. 337.

${ }^{29}$ Directiva 2001/18/CE.
} 
El Reglamento $1830 / 2003^{30}$ en su Considerando 3, fija los tres objetivos básicos de la trazabilidad de los OMG, a saber:

1. Facilitar la aplicación de las medidas de gestión del riesgo de acuerdo con el Principio de Precaución.

2. Permitir el seguimiento selectivo de los posibles efectos sobre el medio ambiente.

3. Facilitar la retirada de productos ante efectos adversos imprevistos sobre la salud humana, la sanidad animal o el medio ambiente.

En síntesis, la trazabilidad de los OMG es una obligación que surge de la norma con la finalidad de asegurar la inocuidad de los mismos y facilitar la detección de potenciales riesgos que puedan producirse a posteriori de su introducción en el mercado y su consecuente retirada.

Asimismo, el Reglamento CE 178/2002 en su Art. 18, establece que en todas las etapas de la producción, transformación y distribución deberá asegurarse la trazabilidad de alimentos, piensos, animales destinados a la producción de alimentos y cualquier otra sustancia destinada a ser incorporada en un alimento o un pienso.

La Ley 17/2011, por su parte, establece en su Art. 6 un requisito de similares características al contenido en el Art. 18 del Reglamento CE 178/2002, pero además, incorpora la obligación de que los alimentos y piensos estén adecuadamente etiquetados o identificados a efectos de facilitar su trazabilidad.

\subsection{Em relación con el etiquetado}

La Constitución Española en su Art. 51.1 garantiza la defensa y protección de la seguridad, la salud, y los legítimos intereses económicos de los consumidores y usuarios por parte de los poderes públicos.

Establece firmemente además, en el apartado subsiguiente, la promoción de la información y educación de aquellos.

Estos verdaderos principios rectores fueron recogidos, en aras de la consagración de los derechos de los consumidores y usuarios, por el Art. 8 del Texto Refundido de la Ley General para la Defensa de los Consumidores y Usuarios y otras leyes complementarias ${ }^{31}$, precepto que enumera los denominados derechos básicos de los consumidores y usuarios.

Entre estos derechos se hallan la protección contra los riesgos que pueden afectar su salud o seguridad, y el acceso a información correcta sobre diferentes bienes o servicios.

Podemos admitir, entonces, que el sustento jurídico subyacente del deber de etiquetado de los productos en general, y en particular de los alimentos modificados genéticamente, como manifestación del deber-derecho de protección contra riesgos a la salud

\footnotetext{
${ }^{30}$ REGLAMENTO (CE) No 1830/2003 DEL PARLAMENTO EUROPEO Y DEL CONSEJO de 22 de septiembre de 2003 relativo a la trazabilidad y al etiquetado de organismos modificados genéticamente y a la trazabilidad de los alimentos y piensos producidos a partir de éstos, y por el que se modifica la Directiva 2001/18/CE.

${ }^{31}$ En adelante TRLGDCU.
} 
y de información, se halla, canalizándose a través del Art. 8 TRLGDCU, en la letra de la propia Constitución.

A diferencia del tratamiento de la cuestión en el sistema de responsabilidad por productos en los Estados Unidos, el legislador europeo ha prescindido de la tipología de los defectos, estableciendo un concepto unitario de defecto basado exclusivamente en el déficit en la seguridad que el público puede esperar legítimamente respecto de los productos.

Sin embargo, existe una serie de defectos que, de alguna manera, han sido autónomamente valorados por el concepto legal de producto defectuoso. Estos defectos son los de información, expresamente considerados por el Art. 137 del TRLGDCU al hacer referencia a la "presentación del producto" como factor especialmente a tener en cuenta para determinar el defecto del mismo ${ }^{32}$.

Fue la preocupación de los consumidores frente al consumo de productos alimenticios compuestos por OMG y su demanda de una rotulación apropiada que les permitiese distinguir y escoger entre productos de esta clase y convencionales ${ }^{33}$, la que motivó el dictado de los Reglamentos 1829/2003 sobre alimentos y piensos modificados genéticamente; y el 1830/2003 relativo a la trazabilidad y al etiquetado de organismos modificados genéticamente y a la trazabilidad de los alimentos y piensos producidos a partir de éstos, y por el que se modifica la Directiva 2001/18/CE.

En materia específica de OMG, existen dos etapas temporalmente distintas donde la información adquiere relevancia sustancial.

La primera de ellas es la fase de trámites de autorización para la producción y comercialización de OMG en un territorio determinado. El objetivo prioritario en esta etapa consiste en otorgar mayor transparencia en los procedimientos administrativos de autorización, a fin de reafirmar la confianza de los consumidores en las autoridades que controlan dicho proceso.

La segunda etapa se inicia luego de la autorización administrativa, al momento de la comercialización de los productos, y consiste en el ofrecimiento al consumidor final de datos precisos e inteligibles sobre la presencia de OMG en los mismos. En este caso, la finalidad perseguida es doble: por un lado permitir al consumidor el acceso al conocimiento sobre qué

\footnotetext{
32 En relación a productos farmacéuticos se había pronunciado el Tribunal Supremo en un sentencia del año 2010 en el sentido de que "la responsabilidad del fabricante puede basarse en el lanzamiento al mercado, bien de productos defectuosos bien de productos que, sin serlo, no aparecen acompañados de las debidas instrucciones para el correcto uso" (SAP de León, (Sección 1ạ), núm. 459/2010 de 29 noviembre - AC 2011/713). Disentimos con este criterio que considera al producto comercializado sin las instrucciones debidas como un producto no defectuoso, y entendemos que el mismo se encontraba ya por entonces legislativamente superado considerando que, a partir de las disposiciones del Art. 3 de la Ley 22/1994 (aplicada al caso que estudiamos) ya se hallaba establecido que aquel producto que adolecía de un déficit de información sería considerado un producto defectuoso, dado que entre las circunstancias que se debían tener en cuenta para su consideración, se encontraba expresamente prevista la presentación del mismo.

33 "En las encuestas que hemos realizado, el 95\% de los consumidores lo ha pedido (la indicación del carácter de transgénico en el envase del producto) porque quiere ejercer su derecho a elegir, explica Gema Trigueros, del departamento técnico de la Organización de Consumidores y Usuarios (OCU). El que el producto esté etiquetado garantiza este derecho [...]" EL MUNDO. 2004: Nuevas exigencias de etiquetado de los alimentos transgénicos. Disponible en: http://www.elmundo.es/elmundosalud/2004/04/19/dieta/1082357695.html. Acesso em 3 abr. 2010.
} 
productos en el mercado son genéticamente modificados y cuáles no y, por el otro, facilitar la libertad de elección entre productos genéticamente modificados o sus análogos convencionales, evitando a los consumidores incurrir en error ${ }^{34}$.

El Reglamento 1830/2003 establece la obligación de que todos los productos transgénicos, en todas las fases de su comercialización hasta llegar al consumidor final, indiquen claramente su carácter de transgénico, mediante documentos escritos entre operadores, y en el etiquetado del producto en relación a los consumidores. El objetivo que se persigue es poder seguir el rastro del transgénico a través de todas sus etapas de producción y distribución.

De acuerdo con lo dispuesto en el Art. 4.6 de la mencionada norma, en el caso de los productos que contienen o están compuestos por OMG, los operadores garantizarán que:

1. Para los productos preenvasados que contienen o están compuestos por OMG, en la etiqueta constará la indicación "Este producto contiene organismos modificados genéticamente", o bien "Este producto contiene (nombre del o de los organismos) modificado(s) genéticamente";

2. Para los productos no preenvasados ofrecidos al consumidor final, la indicación "Este producto contiene organismos modificados genéticamente" o "Este producto contiene (nombre del o de los organismos), modificado(s) genéticamente" constará en la presentación del producto o en los elementos asociados a dicha presentación.

Por su parte, el Reglamento $1829 / 2003$, establece que todo alimento que contenga o esté compuesto por OMG, o se haya producido a partir de OMG o contenga ingredientes producidos a partir de estos organismos, está sujetos a los siguientes requisitos de etiquetado:

1. Si el alimento está compuesto por más de un ingrediente, figurará entre paréntesis, inmediatamente después del ingrediente en cuestión, el texto "modificado genéticamente" o "producido a partir de (nombre del ingrediente) modificado genéticamente".

2. Si el ingrediente viene designado por el nombre de una categoría, en la lista de ingredientes figurará el texto "contiene (nombre del organismo) modificado genéticamente" o "contiene (nombre del ingrediente) producido a partir de (nombre del organismo) modificado genéticamente".

3. A falta de una lista de ingredientes, en el etiquetado figurará claramente el texto "modificado genéticamente" o "producido a partir de (nombre del organismo) modificado genéticamente".

4. Si el alimento se ofrece para su venta al consumidor final como alimento no preenvasado o como alimento preenvasado en pequeños recipientes cuya mayor superficie consiste en un área de menos de $10 \mathrm{~cm}^{2}$, la información exigida en el presente apartado deberá exhibirse visible y permanentemente, bien en el expositor del alimento, bien inmediatamente al lado, o en el envase, en un tipo de letra lo suficientemente grande para su fácil identificación y lectura.

Además de los requisitos enumerados, si el alimento es diferente a su homólogo convencional en cuanto a su composición, valor o efectos nutricionales, uso para el que está

${ }^{34}$ AMAT LLOMBART, P. Op. cit., 158-159. 
destinado o repercusiones para la salud de determinados sectores de la población, la etiqueta deberá mencionar las características o propiedades especificadas en la autorización.

Igual requisito en el etiquetado es exigido cuando el alimento pueda generar inquietudes de orden ético o religioso.

Cualquier etiquetado tiene como objetivo ofrecer a los consumidores información sobre el producto que no sería posible identificar a simple vista, teniendo como consecuencia no sólo la detección de la presencia de OMG en el producto, sino también la posibilidad de que el consumidor pueda elegir con conocimiento de causa y libertad, como se ha expresado previamente.

Es así que en lo relativo a quiénes sean los destinatarios de la información con la que el productor debe presentar el producto, los tribunales exigen siempre una información dirigida al público consumidor en general, incluso respecto a productos que usualmente sólo serán administrados o prescritos por profesionales o expertos. Aún en estos supuestos, se impone al fabricante el deber de proporcionar una información destinada al público consumidor en general.

La etiqueta no debe ser concebida como un instrumento de advertencia sanitaria negativa o disuasoria al consumidor, sino que está pensada para permitirle llevar a cabo su opción de compra libremente.

En relación con la seguridad de los alimentos modificados genéticamente, cabe señalar que las evaluaciones sanitarias que deben superar (en la UE) son mucho más rigurosas que las que debe cumplir cualquier otro tipo de alimento, aunque las mismas no se encuentran exentas de críticas (explican FAGAN et al. que el proceso de evaluación europeo, aunque más fiable que el de los Estados Unidos, no consiste en un sistema riguroso para evaluar el potencial alergénico de los alimentos modificados genéticamente, aparte de no ser un proceso de evaluación objetivo al haber sido generado por instituciones vinculadas al sector productor agrobiotecnológico) $)^{35}$.

Por ello, la normativa sobre etiquetado obligatorio de este tipo de alimentos no constituye un instrumento de alarma, ni de castigo hacia ellos (como lo entienden determinados lobbies principalmente en los Estados Unidos) sino un vehículo de confianza para la sociedad y para el libre ejercicio de los derechos de los consumidores ${ }^{36}$.

Para la jurisprudencia, la relevancia de los defectos de información radica en la presunción de que, de haberse proporcionado la información adecuada, hubiera podido evitarse el daño sufrido, habiendo llegado los tribunales a considerar defectuosos productos

\footnotetext{
${ }^{35}$ Esto se debe a que no se han desarrollado pruebas científicas fiables para predecir la alergenicidad. El proceso que los reguladores de la UE utilizan para evaluarla se basa en un sistema propuesto en 2001 por la Organización para la Agricultura y la Alimentación (FAO) de las Naciones Unidas y la Organización Mundial de la Salud (OMS), el cual fue diseñado, en realidad, por dos grupos financiados por la industria biotecnológica, el Instituto Internacional de Ciencias de la Vida (ILSI) y el Consejo Internacional de Biotecnología Alimentaria (IFBC). FAO/WHO. 2001: Evaluation of Allergenicity of Genetically Modified Foods. Report of a Joint FAO/WHO Expert Consultation on Allergenicity of Foods Derived from Biotechnology, Roma, 5. http://www.fao.org/3/a-y0820e.pdf [19 de marzo de2015].

36 GUTIERREZ BARRENENGOA, A. y MONJE BALMASEDA, O. El etiquetado de los alimentos modificados genéticamente en el ámbito de la Unión Europea. En Aspectos legales de la agricultura transgénica. Almería: Universidad de Almería- Servicio de Publicaciones, 72-73, 2004.
} 
que sin ser intrínsecamente inseguros, causaron daños por haber sido comercializados sin informar adecuadamente al consumidor sobre su modo de uso y potenciales riesgos, lo que indudablemente generó una considerable discrepancia entre la seguridad esperada del producto y la realmente ofrecida por éste.

No obstante, es conveniente aclarar que no se exonera de responsabilidad al productor por el hecho de haber advertido convenientemente de los riesgos inherentes al producto, si le hubiera sido posible evitarlos incorporando a su diseño algún dispositivo adicional de seguridad. Permitir al productor suplir con información aquellas mejoras que omite aun siéndole técnicamente viables, sería tanto como hacer soportar al consumidor los riesgos derivados de esas deficiencias del producto, deliberadas, o cuando menos, evitables ${ }^{37}$.

\section{EL PRINCIPIO DE PRECAUCIÓN}

El Principio de Precaución es uno de los principios de mayor envergadura en la materia que venimos tratando y tiene receptividad en gran parte de los ordenamientos jurídicos internacionales, ya sea mediante su incorporación a tratados celebrados entre distintos Estados ${ }^{38}$, o mediante su inclusión expresa en normativas locales de seguridad alimentaria y de tutela del medio ambiente ${ }^{39}$.

No existe una definición universalmente aceptada de este principio. Cada una de las elaboradas hace hincapié en uno u otro elemento componente del mismo. Sin embargo, todas coinciden en que nos encontramos ante una acción cautelar, frente a una actividad que despierta la sospecha científicamente sustentada ${ }^{40}$ de poder acarrear un daño grave tanto a la salud del ser humano como al medio ambiente.

37 MARCO MOLINA, J. La responsabilidad civil del fabricante por productos defectuosos. Fundamentos y Aplicación. Barcelona: Atelier, 161, 2007.

${ }^{38} \mathrm{Su}$ primer reconocimiento internacional se plasmó en la Carta Mundial de la Naturaleza, aprobada por la Asamblea General de las Naciones Unidas en 1982, y su consagración en materia de protección y preservación del medio ambiente y la biodiversidad (ámbito de aplicación que luego sería extendido también al área de la salud humana) se produjo a través de su inclusión en la "Declaración de Río sobre el Medio Ambiente y el Desarrollo" (1992) que en su principio 15 establece: "Con el fin de proteger el medio ambiente, los Estados deberán aplicar ampliamente el criterio de precaución conforme a sus capacidades. Cuando haya peligro de daño grave o irreversible, la falta de certeza científica absoluta no deberá utilizarse como razón para postergar la adopción de medidas eficaces en función de los costos para impedir la degradación del medio ambiente." Asimismo, fue incorporado a la Convención sobre la Diversidad Biológica y a la Convención de Cambio Climático, aprobadas también en la Conferencia de Río. Posteriormente, en el año 2000, el "Protocolo de Cartagena sobre seguridad de la biotecnología" incluyó el Principio de Precaución como uno de sus objetivos, a través del cual se admite que un país pueda negarse a la importación de OMG alegando solamente dudas sobre la seguridad de ese producto para la salud o el medio ambiente (Art. 1, firmado en enero de 2000 en Montreal, entrando en vigor para las partes, entre ellas la UE, el 13 de septiembre de 2003).

${ }^{39}$ En la mayoría de los instrumentos internacionales el principio de precaución se presenta como un principio poco vigoroso e impreciso, con escaso desarrollo como para permitir su aplicación uniforme en el plano global, pero en la práctica interna de los Estados hay una creciente tendencia a incorporarlo con rango constitucional y a dotarlo de herramientas suficientes para su aplicación en concreto.

40 “En tal sentido, resulta ilustrativo señalar que el órgano de apelación del Sistema de Solución de Diferencias de la OMC considera que el principio de cautela que rige en materia fitosanitaria, no es suficiente para relevar a 
La Cláusula Delaney introdujo hace algunos años un sistema de interpretación jurídica ante la incertidumbre en el ordenamiento jurídico de los Estados Unidos que puede considerarse como un antecedente de este principio.

Esta fórmula fue incorporada en el año 1958 en la Ley Federal de Alimentos, Medicamentos y $\operatorname{Cosméticos}^{41}$ del país norteamericano, disponiendo que un aditivo no se considerará seguro (y, por lo tanto, su empleo estará prohibido) si se prueba que puede provocar cáncer cuando es ingerido por el hombre o los animales. Dicha cláusula consagró, por lo tanto, el criterio de tolerancia $\operatorname{cero}^{42} \mathrm{y}$, en principio, se aplicaba igualmente a los residuos de plaguicidas. La adopción de dicha cláusula fue promovida por un congresista llamado Delaney, que durante los años cincuenta presidió una comisión parlamentaria de investigación sobre el uso de productos químicos en los alimentos, y uno de cuyos resultados desató una campaña en los medios y opinión pública sobre la seguridad alimentaria. Desde sus orígenes se puso en entredicho la eficacia de esta cláusula tanto desde la perspectiva científica como jurídica.

En la actualidad, nadie apuesta seriamente por el mantenimiento de dicha cláusula, que ha ido siendo sustituida progresivamente por la noción de "certeza razonable de ausencia de daño" ${ }^{\prime 3}$.

Hoy en día se considera que para el progreso en el campo biotecnológico no existe la idea de riesgo cero, por lo cual surge el deber de proteger y regular las actuaciones de la biotecnología que puedan llegar a producir daños. Ello se materializa, por ejemplo, a través del recurso al principio de precaución.

La doctrina reconoce que las primeras manifestaciones del principio de precaución se encuentran en Alemania en los albores de los años setenta, bajo la denominación de Vorsorgeprinzip, como consecuencia de la toma de conciencia de que determinados químicos podrían tener consecuencias muy negativas y ante la incertidumbre acerca del efecto de tales sustancias, el Vorsorgeprinzip impulsaba a actuar a pesar de la incertidumbre o en virtud de ella ${ }^{44}$.

El sector de la biotecnología es el terreno de principal funcionamiento del Principio de Precaución, por cuanto éste es llamado a operar en situaciones de incertidumbre científica, legitimando una intervención defensiva incluso cuando la existencia misma del peligro es dudosa, momento precedente respecto a aquel al cual, normalmente, es subordinada una intervención de tipo preventivo (la certeza de la existencia del peligro) ${ }^{45}$.

Así lo manifestó la propia Comisión Europea en su Comunicación de 2 de febrero de 2000, en la que literalmente expresa que este principio "abarca los casos específicos en los que los datos científicos son insuficientes, no concluyentes o inciertos, pero en los que existe evaluación científica objetiva preliminar que hace sospechar que existen motivos razonables

\footnotetext{
la parte que establece restricciones a la importación de la prueba científica de la argumentación”. COZZI, E. H. 2005: El principio de precaución. Las patentes de invención y la responsabilidad civil. Buenos Aires: Ad-Hoc, 62.

${ }^{41}$ Federal Food, Drug and Cosmetic Act.

42 PÉREZ, M. I. La manzana de Blancanieves. Salud, industria y alimentación. Barcelona: Icaria Editorial, 24, 2006.

${ }^{43}$ Original en inglés: reasonable certainty of no harm.

${ }^{44}$ HERRERA DE LAS HERAS, R. La responsabilidad derivada de los daños producidos por la biotecnología. Madrid: Reus, 43-44, 2007.

${ }^{45}$ CASONATO C. y BERTI M. II Diritto degli OGM tra Possibilità e Scelta. Atti del Convegno tenuto presso la Facoltà di Giurisprudenza di Trento, 26 novembre 2004. Trento: Ed. Litotipografia Alcione, 62, 2006.
} 
para temer que los efectos potencialmente peligrosos para el medio ambiente y la salud humana, animal o vegetal, pudieran ser incompatibles con el alto nivel de protección elegido".

Por otra parte, dentro del sistema del Common Law, se entiende que la aplicación del principio de precaución es de alta sensibilidad ya que, generalmente, choca con ciertas libertades individuales y el libre comercio; particularmente, con este último, principio ya consolidado en el sistema global. Los doctrinarios del Common Law sostienen que el principio es aplicable de modo limitado, proporcional a los riesgos temidos, aplicado de modo no discriminatorio, basado en la relación costo-beneficio, y siempre que no vulnere libertades individuales ${ }^{46}$.

En este marco, Estado Unidos, que ostenta un lugar de relevante poder político en el ámbito del comercio mundial, afronta las situaciones de incertidumbre científica de manera diversa a la UE ${ }^{47}$. "En una situación de duda sobre los posibles efectos derivados de una sustancia o de un producto, es decir, en presencia de un mero riesgo potencial, los Estados Unidos eligen no intervenir, no poner vetos, hasta que la ciencia no esté en grado de dar respuestas ciertas; sólo entonces, eventualmente, el poder público adoptará contramedidas" 48 .

En la actualidad, sin embargo, si bien las referencias explícitas a este principio en la legislación norteamericana son escasas, existe un conjunto de normas ${ }^{49}$ y declaraciones que de alguna manera lo presuponen ${ }^{50}$, y se ha empezado a detectar una creciente tendencia hacia su implementación ${ }^{51}$.

\footnotetext{
${ }^{46}$ DRNAS DE CLEMENT Z. Op. cit., 22.

${ }^{47}$ Este país se opone a la adopción del Principio de Precaución al considerarlo un impedimento para el desarrollo y expansión del comercio internacional. No ha ratificado la Convención de Río sobre Bioseguridad y, junto a Canadá, desconocen su carácter de norma de derecho internacional. COZZI, E. H. Op.cit, 67. Esta situación fue constatada en el año 2003, cuando Estados Unidos realizó una serie de consultas (junto a Argentina y Canadá) ante la OMC, en relación a medidas adoptadas por la UE vinculadas a la aprobación y comercialización de productos biotecnológicos que afectaban a la importación de productos agropecuarios y alimenticios procedentes de los tres países. En el informe surgido de esta controversia y presentado por la OMC el 29 de septiembre de 2006 Estados Unidos manifestó que "el principio de precaución o cautela no puede ser considerado un principio general ni una norma de derecho internacional porque no tiene una formulación única y convenida [...] Por consiguiente, los Estados Unidos consideran que la precaución o cautela es un enfoque y no un principio del derecho internacional". DE ROMÁN PEREZ, R. Op. cit., 364. Concluyó Estados Unidos su exposición manifestando su falta de acuerdo en que se recurra al Protocolo sobre Seguridad de la Biotecnología para interpretar el Acuerdo de la OMC en cuestión, ni siquiera en una diferencia entre dos miembros de la OMC suscriptores del Protocolo. OMC, COMUNIDADES EUROPEAS. Medidas que afectan a la aprobación y comercialización de productos biotecnológicos. Informes del Grupo Especial. Reclamaciones WT/DS291/R, WT/DS292/R y WT/DS293/R, Segunda Comunicación escrita de los Estados Unidos.

${ }^{48}$ CARRETERO GARCÍA, A. 2006: Algunas consideraciones sobre el Principio de Precaución como instrumento de protección de la salud de los consumidores en el ámbito alimentario. Disponible en: https://www.uclm.es/centro/cesco/pdf/trabajos/10/2006/10-2006-1.pdf, p. 6, Acesso en: 5 jul. 2015.

${ }^{49}$ The U.S. Toxic Substances Control Act, the Food Quality and Protection Act of 1996, The National Environmental Policy Act, the Endangered Species Act, the Clean Water Act, entre otras.

50 DE CÓZAR ESCALANTE, J. M. 2005: "Principio de Precaución y medio ambiente", Rev. Esp. Salud Pública, V.79, № 2.

51 "The precautionary principle, virtually unknown here six years ago, is now a U.S. phenomenon. In December 2001 the New York Times Magazine listed the principle as one of the most influential ideas of the year" (EI Principio de Precaución, virtualmente desconocido en este país hace seis años, es actualmente un fenómeno en los Estados Unidos. En Diciembre del 2001 la revista New York Times lo enumeró como una de las ideas más
} 
A nivel global, el Convenio sobre la Diversidad Biológica, aprobado el 5 de junio de 1992 en el marco de la Conferencia de Naciones Unidas sobre el Medio Ambiente y el Desarrollo, y ratificado por España el 16 de noviembre de 1993, define a la biotecnología y establece en su Art. 19 (donde regula específicamente los aspectos vinculados a esta actividad) la necesidad de establecer un Protocolo de procedimientos adecuados en la esfera de la transferencia, manipulación y utilización de OMG. Esta reunión celebrada en Río de Janeiro, Brasil, del 3 al 14 de junio de 1992, presenta como uno de sus triunfos más salientes la Declaración de Río sobre Medio Ambiente y Desarrollo. La misma tiene como logro principal dentro del ámbito que nos ocupa y dentro de su Principio $15^{52}$, la consagración expresa del Principio de Precaución.

A raíz de la inclusión del Principio de Precaución en el Protocolo de Cartagena en el año 2000, se dejaron expuestas las divergentes posiciones al respecto entre la Unión Europea y el Grupo de Miami (encabezado por Estados Unidos, Canadá y Argentina, y que aglutinaba a los mayores productores de cultivos transgénicos) generándose controversias con la OMC en relación a las normas sobre libre comercio mundial ${ }^{53}$.

Luego de lo expuesto, se divisa claro el real panorama de operatividad del Principio de Precaución en los distintos sistemas legales expuestos, panorama que SPROVIERI y DELL'OCA sintetizan de la siguiente manera: "Con todo, un fantasma aparece una y otra vez entre quienes defienden el principio de precaución. Sólo algunos lo ponen honestamente negro sobre blanco. Ese fantasma no es otro que los intereses económicos que mediante la hegemonía del crudo criterio costo-beneficio, que finalmente sólo satisface en forma inmediata el interés económico de las empresas, pone en riesgo con nuevas tecnologías la salud pública y el medio ambiente" ${ }^{\prime 54}$.

\subsection{Materialización del Principio de Precaución}

Desde el punto de vista de su ejecución por parte de la Administración, las medidas derivadas del recurso al principio de precaución pueden adoptar la forma de una decisión de actuar o de no actuar. La respuesta escogida depende de una decisión política, en función del nivel de riesgo considerado "aceptable" por la sociedad que debe soportarlo ${ }^{55}$.

Es así que las autoridades cuentan con un amplio abanico de acciones entre las que elegir al momento de recurrir al principio de precaución, como son la financiación de programas de investigación, o proceder a informar a la opinión pública sobre los efectos

\footnotetext{
influyentes del año", SCIENCE \& ENVIRONMENTAL HEALTH NETWORK. (s.f.): Precautionary Principle. Disponible en: http://www.sehn.org/precaution.html. Acesso en: 24 jul. 2015.

52 Principio 15 - Declaración de Río sobre Medio Ambiente y Desarrollo: “Con el fin de proteger el medio ambiente, los Estados deberán aplicar ampliamente el criterio de precaución conforme a sus capacidades. Cuando haya peligro de daño grave o irreversible, la falta de certeza científica absoluta no deberá utilizarse como razón para postergar la adopción de medidas eficaces en función de los costos para impedir la degradación del medio ambiente."

53 OMC, COMUNIDADES EUROPEAS. Medidas que afectan a la aprobación y comercialización de productos biotecnológicos. Informes del Grupo Especial. Reclamaciones WT/DS291/R, WT/DS292/R y WT/DS293/R.

54 SPROVIERI, L. E. y DELL'OCA, G. Daños por Productos Elaborados. Buenos Aires: Abeledo Perrot, 482, 2012.

${ }^{55}$ Comunicación de la Comisión, de 2 de febrero de 2000, sobre el recurso al principio de precaución [COM (2000) $1]$.
} 
potencialmente peligrosos de un producto o de un método. El recurso a este principio no se traduce necesariamente en la aprobación de normas o reglamentos destinados a producir efectos jurídicos.

Estas medidas deberán mantenerse en vigencia mientras los datos científicos sigan siendo insuficientes, y mientras el riesgo se considere lo suficientemente alto como para no aceptar que la sociedad deba asumirlo. Pero ante la existencia de nuevos datos y avances científicos, las medidas deberán ser modificadas o suprimidas.

\subsection{El Principio de Precaución en la UE}

Según DOMÉNECH PASCUAL ${ }^{56}$, es en el Tratado de Maastricht de 1992, donde asoma por vez primera este principio como tal (disociado de la idea de prevención y mediante la voz "cautela") en la legislación comunitaria ${ }^{57}$.

Posteriormente, la Comunicación de la Comisión sobre el recurso al Principio de Precaución de 2 de febrero de 2000, demuestra inequívocamente la presencia de este principio en la normativa europea estableciendo que: "La Comisión considera que la Comunidad, al igual que otros miembros de la OMC, tiene derecho a establecer el nivel de protección que considere adecuado, en particular en lo que se refiere al medio ambiente y la salud humana, animal o vegetal. La aplicación del principio de precaución constituye un principio esencial de su política [...]"

A su vez, la Directiva 2001/18/CE estableció que "los Estados miembros garantizarán, de conformidad con el principio de cautela, la adopción de todas las medidas adecuadas para evitar efectos negativos sobre la salud humana y en el medio ambiente que pudieran resultar de la liberación intencional o de la comercialización de $\mathrm{OMG}^{59}$ y que "se ha tenido en cuenta el principio de cautela a la hora de redactar la presente Directiva y debe tenerse en cuenta cuando ésta se aplique ${ }^{60 \prime \prime}$.

Pero uno de los documentos de mayor importancia en cuanto a la definición del principio de precaución es el Reglamento (CE) No 178/2002, especialmente en su Art. 7, el cual dispone en su apartado 2 que: "En circunstancias específicas, cuando, tras haber evaluado la información disponible, se observe la posibilidad de que haya efectos nocivos para la salud, pero siga existiendo incertidumbre científica, podrán adoptarse medidas provisionales de gestión del riesgo para asegurar el nivel elevado de protección de la salud por el que ha optado la Comunidad, en espera de disponer de información científica adicional que permita una determinación del riesgo más exhaustiva".

\footnotetext{
56 DOMÉNECH PASCUAL, G. Op. cit., 276.

${ }^{57}$ Tratado de la Unión Europea, art 130 R.2: "La política de la Comunidad en el ámbito del medio ambiente tendrá como objetivo alcanzar un nivel de protección elevado, teniendo presente la diversidad de situaciones existentes en las distintas regiones de la Comunidad. Se basará en los principios de cautela y de acción preventiva, en el principio de corrección de los atentados al medio ambiente, preferentemente en la fuente misma, y en el principio de quien contamina paga."

${ }^{58}$ Comunicación de la Comisión sobre el recurso al principio de precaución de 2 de febrero de 2000, apartado 3 del Resumen.

59 Directiva 2001/18/CE, Art. 4.1.

${ }^{60}$ Directiva 2001/18/CE, Considerando (8).
} 
En el Asunto C-6/99 Association Greenpeace France y otros / Ministère de l'Agriculture et de la Pêche y otros, el TJCE respaldó en forma expresa el Principio de Precaución disponiendo que aun ante a la existencia de una decisión favorable por parte de la Comisión como respuesta a una petición de comercialización de un organismo modificado genéticamente "si el Estado miembro afectado dispone mientras tanto de nuevos elementos de información que le llevan a considerar que el producto que ha sido objeto de la notificación puede presentar un riesgo para la salud humana y el medio ambiente, no estará obligado a dar su autorización, siempre y cuando lo comunique inmediatamente a la Comisión y a los demás Estados miembros".

Similar conclusión adoptó el Tribunal de Primera Instancia de las Comunidades Europeas en la sentencia "Les Laboratoires Servier", en la que precisó que "si disponen de datos nuevos (con respecto a la última evaluación de los medicamentos considerados [...]) que, aun sin disipar la incertidumbre científica, permiten razonablemente dudar de la inocuidad y/o de la eficacia de tales medicamentos y conducen a una apreciación desfavorable de su balance riesgos/beneficios, las autoridades competentes están obligadas, en virtud del artículo 11 de la Directiva 65/65 ${ }^{61}$ interpretado en relación con el principio de cautela, que constituye un principio general del Derecho comunitario, a suspender o retirar la [autorización de comercialización] de dichos medicamentos"62.

\subsection{El Principio de Precaución en España}

La Ley 9/2003, se ve inspirada por el Principio de Precaución y Cautela, disponiendo expresamente en su Exposición de Motivos que el mismo implica adoptar las medidas adecuadas para evitar los potenciales efectos adversos para la salud humana y el medio ambiente derivados de estas actividades.

Por su parte, la Ley 17/2011, en su Art. 7 prevé que "en circunstancias específicas, y en particular ante la aparición de riesgos emergentes, cuando tras haber evaluado la información disponible, se observe la posibilidad de que haya efectos nocivos para la salud, pero siga existiendo incertidumbre científica, podrán adoptarse medidas provisionales de gestión del riesgo para asegurar la protección de la salud, todo ello en espera de una información científica adicional, que permita una evaluación del riesgo más exhaustiva".

Asimismo, determina esta última norma que dichas medidas deberán ser revisadas en un tiempo razonable, conforme el riesgo contemplado y la información científica adicional para aclarar la incertidumbre y llevar a cabo una evaluación del riesgo más exhaustiva.

\footnotetext{
${ }^{61}$ Directiva 65/65/CEE del Consejo, de 26 de enero de 1965, relativa a la aproximación de las disposiciones legales, reglamentarias y administrativas, sobre especialidades farmacéuticas, Art. 11: "Las autoridades competentes de los Estados miembros suspenderán o retirarán la autorización de comercialización cuando la especialidad farmacéutica resulte ser nociva en las condiciones normales de empleo, cuando carezca de efectos terapéuticos o finalmente cuando no posea la composición cualitativa y cuantitativa declarada ".

${ }^{62}$ GONZÁLEZ VAQUÉ, L. El Principio de Precaución en la Jurisprudencia del Tribunal de Justicia de las Comunidades Europeas: ¿Un principio de buen gobierno?, N. 16, 2004. Disponible en: http://consumoinc.gob.es/publicac/EC/2004/EC68/EC68_01.pdf. Acesso en: 24 jul. 2015.
} 


\section{EL PRINCIPIO DE PREVENCIÓN}

Este principio descansa sobre las bases de la actuación preventiva ante la certeza de un riesgo. Procede su aplicación cuando existe seguridad acerca de su existencia.

El Principio de Prevención, está asentado en la idea de "diligencia debida" (deber jurídico), es decir, en la obligación de vigilancia y adopción de previsiones en relación a los bienes y personas bajo su jurisdicción, a fin de asegurarse que, en condiciones normales, los objetos, elementos o actividades riesgosas no causen perjuicios a terceros ${ }^{63}$.

La diferencia esencial con el Principio de Precaución está dada, fundamentalmente, por el carácter del riesgo que motiva su aplicación, ya que en este último el riesgo es dudoso, no se conoce con certeza, simplemente es posible.

El Principio de Precaución, en opinión de un importante sector de la doctrina, se basa en el "buen gobierno", gestión que se adelanta a los hechos, la que ante la duda de que una actividad pueda ser riesgosa, prefiere limitarla (aun equivocándose), privilegiando las seguridades. En el caso de sospecha de riesgo de daño grave, irreversible o catastrófico, la no adopción de medidas cautelares genera responsabilidad similar a la de la falta de prevención si el daño llega a producirse ${ }^{64}$.

El Principio de Prevención, por su parte, es el resultado de una política de confianza en la ciencia y reglamentación de mínimos para cumplir con los requisitos marcados por ésta, protegiendo bienes jurídicos concretos de daños concretos.

En suma, al riesgo verificado corresponde la prevención; al riesgo potencial, la precaución.

\section{CONSIDERACIONES FINALES}

Nos inclinamos por conceptualizar al riesgo como un peligro o amenaza connatural al sistema tecnoindustrial global. En este contexto, somos conscientes, siguiendo a CARR e IBARRA, de que el riesgo cero no existe ${ }^{65}$, y menos aún en las actividades y productos propios de estos tiempos, como es la producción y consumo de OMG, de consecuencias, hasta el momento, indeterminadas.

Así, podemos identificar dos maneras de gestionar estos nuevos riesgos: una, mediante el cálculo de probabilidades y la consecuente asunción de la responsabilidad por el riesgo efectivamente producido; y la otra, constituida sobre la idea de cautela y de prevención anticipada.

Dada la gran trascendencia y desarrollo que, en este contexto, ha adquirido la producción de OMG a escala mundial en los últimos tiempos (la superficie agrobiotecnológica ha manifestado un crecimiento sin precedentes, lo cual puso de manifiesto que es la tecnología que ha gozado de la aceptación más rápida en la reciente historia de la agricultura),

\footnotetext{
63 DRNAS DE CLEMENT, Z. Op.cit., 60.

${ }^{64}$ Ibídem.

${ }^{65}$ CARR, S. e IBARRA, A. Op. cit., 61.
} 
y considerando el elevado grado de masividad y catástrofe que eventuales daños asociados a su consumo podrían generar, entendemos que esta nueva especie de riesgo, que parte de la indeterminación del conocimiento científico, requiere, necesariamente, la consagración de la idea de precaución, y es en este sentido que consideramos imprescindible la efectiva operatividad de los principios jurídicos que hemos desarrollado en este trabajo.

Y ello, porque hoy se evidencia la necesidad imperiosa de una nueva construcción y regulación del riesgo que reúna teorías científicas y técnicas para la comprensión y gestión del mismo, pero que, indefectiblemente, incorpore también los valores e intereses de la sociedad contemporánea, la que ha constituido el problema de la seguridad en materia de alimentos en una exigencia prioritaria.

\section{REFERÊNCIAS}

AMAT LLOMBART, P. Derecho de la Biotecnología y los Transgénicos (Especial referencia al sector agrario y alimentario). Valencia: Tirant lo Blanch, 2008.

ARGENBIO - CONSEJO ARGENTINO PARA LA INFORMACIÓN Y EL DESARROLLO DE LA BIOTECNOLOGÍA. (s.f.): Alimentos transgénicos, ¿deberían etiquetarse?. Cuaderno № 14.

Disponible en:

http://www.porquebiotecnologia.com.ar/index.php?action=cuaderno\&opt=5\&tipo=1\&note =14. Acesso en: 25 may. 2010.

CARR, S. e IBARRA, A. Las construcciones del riesgo. En LUJÁN, J. L. y ECHEVERRÍA, J., (Eds.): Gobernar los riesgos. Ciencia y valores en la sociedad del riesgo. Madrid: Ed. Biblioteca Nueva, 2009.

CARRETERo GARCíA, A. 2006: Algunas consideraciones sobre el Principio de Precaución como instrumento de protección de la salud de los consumidores en el ámbito alimentario.

Disponible en: https://www.uclm.es/centro/cesco/pdf/trabajos/10/2006/10-2006-1.pdf. Acesso en: 5 jul. 2015.

CASONATO C. y BERTI M. Il Diritto degli OGM tra Possibilità e Scelta. Atti del Convegno tenuto presso la Facoltà di Giurisprudenza di Trento, 26 novembre 2004. Trento: Ed. Litotipografia Alcione, 2006.

COZZI, E. H. El principio de precaución. Las patentes de invención y la responsabilidad civil. Buenos Aires: Ad-Hoc, 2005.

DE CÓZAR ESCALANTE, J. M. Principio de Precaución y medio ambiente, Rev. Esp. Salud Pública, V.79, № 2, 2005.

DE ROMÁN PÉREZ, R. Organismos modificados genéticamente en el marco del desarrollo rural sostenible. En VATTIER FUENZALIDA, C. (Dir.) y DE ROMÁN PÉREZ, R. (Coord.), EI Desarrollo Sostenible en el Ámbito Rural. Navarra: Thomson Reuters, 2009.

DIAZ COUSELO, J. M. Los Principios Generales del Derecho. Buenos Aires: Ed. Plus Ultra, 1971. DOMÉNECH PASCUAL, G. Derechos Fundamentales y riesgos tecnológicos. Madrid: Centro de Estudios Políticos y Constitucionales, 2006. 
DRNAS DE CLEMENT Z. El principio de precaución ambiental. La práctica argentina. Córdoba: Lerner Editora, 2008.

EL MUNDO. 2004: Nuevas exigencias de etiquetado de los alimentos transgénicos. Disponible en: http://www.elmundo.es/elmundosalud/2004/04/19/dieta/1082357695.html. Acesso en: 3 abr. 2010.

EL PAÍS. Aprobado el primer animal transgénico para consumo humano. Disponible en: http://elpais.com/elpais/2015/11/19/ciencia/1447945426_325310.html. Acesso en: 21 nov. 2015.

FAO/WHO. 2001: Evaluation of Allergenicity of Genetically Modified Foods. Report of a Joint FAO/WHO Expert Consultation on Allergenicity of Foods Derived from Biotechnology, Roma, 5. Disponible en: http://www.fao.org/3/a-y0820e.pdf. Acesso en: 19 mar. 2015.

FAO. Evaluación de la inocuidad de los alimentos genéticamente modificados. Instrumentos para capacitadores, Roma, 11, 2009. Disponible en: http://www.fao.org/3/a-i0110s.pdf. Acesso en: 19 mar. 2015.

FERNÁNDEZ DÍEZ, M. C. y CORRIPIO GIL - DELGADO, M. R. Los conflictos en la política comercial de la Unión Europea: el caso de la aplicación del Principio de Precaución en la normativa comunitaria de Bioseguridad. En DE TOMÁS MORALES, S. y VAQUERO LAFUENTE, E., El día de Europa: La Europa de los 25. Madrid: Universidad Pontificia Comillas, 2005.

GONZÁLEZ VAQUÉ, L. El Principio de Precaución en la Jurisprudencia del Tribunal de Justicia de las Comunidades Europeas: ¿Un principio de buen gobierno?, n. 16, 2004. Disponible en: http://consumo-inc.gob.es/publicac/EC/2004/EC68/EC68_01.pdf. Acesso en: 24 jul. 2015.

GUTIERREZ BARRENENGOA, A. y MONJE BALMASEDA, O. El etiquetado de los alimentos modificados genéticamente en el ámbito de la Unión Europea. En Aspectos legales de la agricultura transgénica. Almería: Universidad de Almería- Servicio de Publicaciones Internacional de Ciencias de la Vida (ILSI) y el Consejo Internacional de Biotecnología Alimentaria (IFBC), 2004.

HERRERA DE LAS HERAS, R. La responsabilidad derivada de los daños producidos por la biotecnología. Madrid: Reus, 2007.

MARCO MOLINA, J. La responsabilidad civil del fabricante por productos defectuosos. Fundamentos y Aplicación. Barcelona: Atelier, 2007.

MOORE, G. y TYMOWSKI, W. Guía Explicativa del Tratado Internacional sobre los Recursos Fitogenéticos para la Alimentación y la Agricultura. UICN Serie de Política y Derecho Ambiental, n. 57, 2008.

PÉREZ, M. I. La manzana de Blancanieves. Salud, industria y alimentación. Barcelona: Icaria Editorial, 2006.

SCIENCE \& ENVIRONMENTAL HEALTH NETWORK. (s.f.): Precautionary Principle. Disponible en: http://www.sehn.org/precaution.html. Acesso en: 24 jul. 2015.

SPROVIERI, L. E. y DELL'OCA, G. Daños por Productos Elaborados. Buenos Aires: Abeledo Perrot, 2012. 